\title{
A Damage Tolerance Model for Plates with Surface Cracks Under Combined End Force and Bending
}

\author{
Yuan Haiyang ${ }^{1,2}$, Wu Yunxin ${ }^{* 1}$ and Liao Zhiqi ${ }^{1}$ \\ ${ }^{I}$ State Key Laboratory for High Performance Complex Manufacturing, College of Mechanical and Electrical \\ Engineering, Central South University, Changsha 410083, China \\ ${ }^{2}$ Hunan College of Information, Changsha 410083, China
}

\begin{abstract}
Reference stress statuses and the critical crack sizes are analyzed systematically and in detail for rectangle surface cracks in plates under combined endforce and cross-thickness bending loads via the 'global' reference stress method. The relationships of critical crack width and depth are obtained from the critical crack sizes analysis. Based on the net-section plastic collapse of the flawed component, a damage tolerance mathematical model for plates with single crack was built, which did not consider the contact of crack faces and it can be used for plates with a shallow crack or a deep crack under combined endforce and cross-thickness bending load. Using this mathematical model, the damage limits of aluminum alloy plates under different bending loads are obtained and a simplified damage tolerance model for aluminum alloy plates is established via regression analysis. The results obtained from the model and the regression model agree well with the experimental results especially when $\mathrm{a} / \mathrm{t}<0.8$. The test results show that the model can be fast and conveniently predict the damage limits for plates with surface cracks under bending.
\end{abstract}

Keywords: Damage tolerance model, net-section plastic collapse, plate, regression analysis, surface cracks.

\section{INTRODUCTION}

With the development of aerospace industry and the ongoing accident occurrence, to accurately predict the damage tolerance and residual life of defect-containing structures has become the key of structure design. The plate structures has been widely used in aircraft structures, such as the whole beam, the whole wall plate, Service performances of plate containing defects such as critical crack size, fatigue crack life and limit load have raised intensive attentions [1-3]. Sattari-Far and Y. Lei etc. respectively analyzed limit loads of plates with surface crack by using local load method and global load method [4-6]. These studies are mainly focused on the limit load of structure containing cracks, while in the process of aircraft structure maintenance and repair, and the engineers could only directly measure the crack size of the structure. It is difficult using limit load to determine whether the structure to be repaired or how long the structure could normally operate. Meanwhile, it is very complicated to use damage tolerance design method to describe fatigue crack propagation and predict the residual life of the structure because of the requirements of a lot of material parameters and the computation of stress intensity factor. This method could be used to calculate propagation and limiting size of two-dimensional penetrating sheet crack. However, as for three-dimensional non-penetrating crack, the process and the calculations are complex while using damage tolerance design method, usually depends on the numerical analysis method to study this kind of crack. The aim of this paper is to establish a unified damage tolerance model which can be used for shallow crack and deep crack.

\section{LOAD AND GEOMETRIC MODEL}

Surface defects of plate type structure are often simplified as half oval or its circumscribed rectangle in plastic failure analysis [7]. The geometric model is shown in Fig. (1). The length is $2 \mathrm{~L}$, the width is $2 \mathrm{~W}$ and the thickness is $t$. In order to make the loading condition of the cracked plate to meet the saint venant principle length must be much bigger than the width and the thickness. A tensile force $\mathrm{N}$ is applied in the center of the end face and a bending moment $\mathrm{M}$ is applied along the through-thickness direction. The tensile force $\mathrm{N}$ in this paper is set as 0 . The crack is assumed to be rectangular-shaped crack as is shown in Fig. (1). The crack is located in the center of the front face with a crack length of $2 \mathrm{c}$ and crack depth of a. Under bending moment, the plate will generate two possible stress distributions. The first one is that the tensile stress is in the front part of the plate and the compressive stress is in the rear part (Fig. 2). A neutral axis separates the two zones. The location of the neutral axial is defined by $\bar{y}$, measured from the front surface of the plate. According to the crack surface, whether by the neutral axial crack, it can be divided into "shallow" crack and "deep" crack is shown in Fig. (2).

In order to facilitate the calculation and promote the scope of the model, the crack depth, length, and the position of the neutral plane are normalized, and are represented by 
$\alpha, \beta$ and $\delta(0<\alpha \leq 1,0<\beta \leq 1,0<\delta \leq 1)$, respectively. The definition is as follows: $\alpha=\frac{a}{t}, \beta=\frac{c}{w}, \delta=\frac{\bar{y}}{t} \cdot \sigma_{b}, \sigma_{m}$ and $\lambda$ are the applied end tensile stresses, bending stresses and the load ratio, defined by $\sigma_{b}=\frac{N}{2 W t}, \sigma_{m}=\frac{3 M}{W t^{2}}$, and $\lambda=\frac{\sigma_{m}}{3 \sigma_{b}}$.
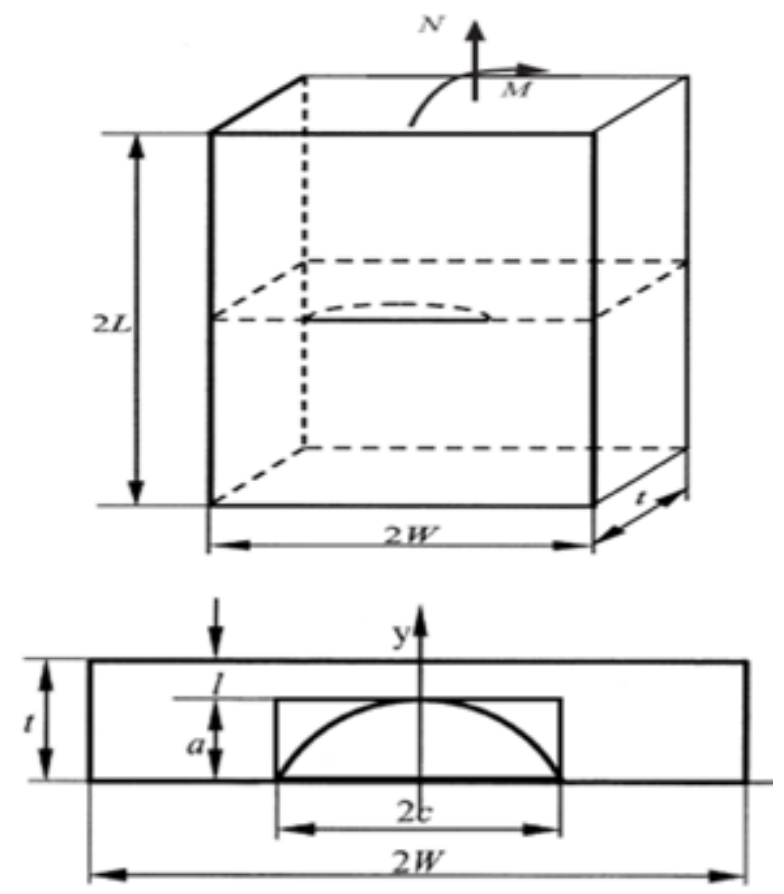

Fig. (1). Schematic of a plate with a surface crack under loads.

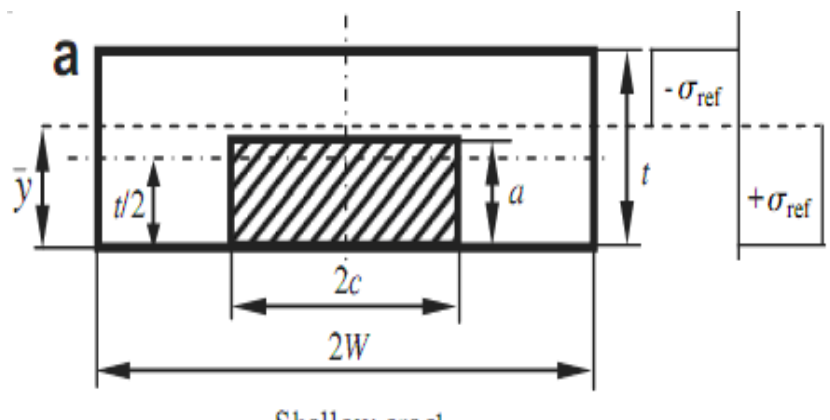

Shallow crack

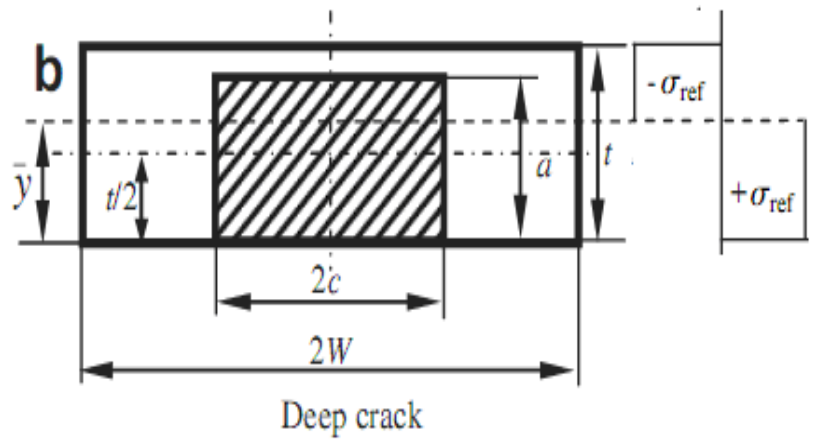

Fig. (2). Assumed stress distribution at plastic collapse under bending.

\section{DAMAGE TOLERANCE ANALYSIS OF CRACKED PLATE UNDER BENDING MOMENT}

The mechanical properties and stress state of the material plays a key role in the initiation and propagation of fatigue crack. Therefore, when calculating crack propagation limit size, the mechanical properties of the material must be known and the analysis of stress state at the crack crosssection must be done. Stress state of the crack section is very complex, there are also a lot of description methods, and reference stress method is adopted in this paper, which can be divided into the global reference stress and the local reference stress [8]. Literature [8-10] has studied the relationship between the reference stress and the corresponding plastic collapse, the relationship between the reference stress and the elastic strain energy and the relationship between the reference stress and stress intensity factor. They confirmed the elastic-plastic fracture mechanics parameter $\mathbf{J}$ and the creep fracture mechanics parameter $\mathrm{C}^{*}$ for flawed components by local reference stress method. Carter AJ studied the limit load of flawed components using the local reference stress [11]. The results of literature [12-14] show that local reference stress is too conservative in the calculation of the crack propagation and the global reference stress could better reflect the characteristics of crack propagation. In this paper, the global reference stress is adopted to analyze the limit crack size of cracked aluminum alloy plate under bending load, and crack closure effects are ignored during the calculation.

\subsection{Limit Damage Analysis for Shallow Cracked Plate}

In shallow cracked section (as in Fig. 2a), to satisfy force and bending moment equilibrium along the direction of the plate length

$$
\left\{\begin{array}{l}
N=2 W \bar{y} \sigma_{r e f}-2 c a \sigma_{r e f}-2 W(t-\bar{y}) \sigma_{r e f} \\
M=W \bar{y} \sigma_{r e f}(t-\bar{y})-c a \sigma_{r e f}(t-a)-W(t-\bar{y}) \sigma_{r e f} \bar{y}
\end{array}\right.
$$

Plug $\alpha, \beta, \delta, \sigma_{b}$ and $\sigma_{m}$ into Eq. (1), can be simplified to

$\left\{\begin{array}{l}\sigma_{b}=\sigma_{r e f}(2 \delta-\alpha \beta-1) \\ \sigma_{m}=3 \sigma_{r e f}\left[2 \delta-2 \delta^{2}-\alpha \beta(1-\alpha)\right]\end{array}\right.$

$\delta$ can be derived from Eq. (2) as follow

$\delta=\frac{\sigma_{b}+\sigma_{r e f}(1+\alpha \beta)}{2 \sigma_{r e f}}$

Using Eq. (3), Eq. (4) can be derived from Eq. (2) as follow

$$
\begin{aligned}
& \sigma_{b}^{2}+2 \sigma_{r e f}\left(\sigma_{b}-\sigma_{r e f}\right)(1+\alpha \beta)+\sigma_{r e f}^{2}(1+\alpha \beta)^{2}-2 \sigma_{b} \sigma_{r e f} \\
& +2 \sigma_{r e f}^{2} \alpha \beta(1-\alpha)+\frac{2}{3} \sigma_{r e f} \sigma_{m}=0
\end{aligned}
$$

In shallow surface crack $0 \leq \alpha \leq \delta$, so $\alpha \leq \frac{\sigma_{b}+\sigma_{r e f}}{\sigma_{r e f}(2-\beta)}$ must be satisfied. The normalized crack length expression for shallow cracks can be derived from Eq. (4) as Eq. (5) with the normalized depth, applied load and reference stress are known. 
Considering $\sigma_{b}>0, \sigma_{m}>0$ and the physically means of shallow crack, the - sign before the square must be forsook, and the + signis the real root of equations (4). When $\sigma_{r e f}$ reaches the yield limit $\sigma_{y}$, the calculated crack size is the limit damage of this structure, the expression is as equations (6).

$$
\beta=\frac{3 \sigma_{r e f}(\alpha-1)-3 \sigma_{b} \pm \sqrt{9 \sigma_{r e f}^{2}\left(\alpha^{2}-2 \alpha+2\right)+6 \sigma_{r e f}\left(3 \sigma_{m}-3 \alpha \sigma_{m}-\sigma_{b}\right)}}{3 \alpha \sigma_{r e f}}
$$

\subsection{Limit Damage Analysis for Deep Cracked Plate}

In shallow cracked section (as in Fig. 2a), to

$\beta=\frac{3 \sigma_{y}(\alpha-1)-3 \sigma_{b}+\sqrt{9 \sigma_{r e f}^{2}\left(\alpha^{2}-2 \alpha+2\right)+6 \sigma_{y}\left(3 \sigma_{m}-3 \alpha \sigma_{m}-\sigma_{b}\right)}}{3 \alpha \sigma_{y}}$

and $\alpha \leq \frac{\sigma_{b}+\sigma_{y}}{\sigma_{y}(2-\beta)}$

satisfy force and bending moment equilibrium along the direction of the plate length

$$
\left\{\begin{array}{c}
N=2 W \bar{y} \sigma_{r e f}-2 c \bar{y} \sigma_{r e f}-2 W(t-\bar{y}) \sigma_{r e f} \\
\quad+2 c(a-\bar{y}) \sigma_{r e f} \\
M=(W-c) \bar{y} \sigma_{r e f}(t-\bar{y})-c(a \\
\quad-\bar{y}) \sigma_{r e f}(a+y-t)-W(t-\bar{y}) \sigma_{r e f} \bar{y}
\end{array}\right.
$$

Plug $\alpha, \beta, \delta$ into Eq. (7) can be simplified to;

$$
\left\{\begin{array}{l}
\sigma_{b}=\sigma_{r e f}(2 \delta+\alpha \beta-2 \beta \delta-1) \\
\sigma_{m}=3 \sigma_{r e f}[2 \delta(1-\delta)(1-\beta)+\alpha \beta(1-\alpha)]
\end{array}\right.
$$

$\delta$ can be derived from Eq. (2) as follow

$\delta=\frac{\sigma_{b}+\sigma_{r e f}(1-\alpha \beta)}{2 \sigma_{r e f}(1-\beta)}$ follow

Using Eq. (9), Eq. (10) can be derived from Eq. (8) as

$$
\begin{aligned}
& \alpha^{2} \sigma_{r e f}^{2} \beta^{2}+2 \sigma_{r e f} \beta\left(\alpha \sigma_{b}-\sigma_{b}-\sigma_{r e f}+\alpha \sigma_{r e f}-\alpha^{2}+\frac{1}{3} \sigma_{m}\right) \\
& -\frac{2}{3} \sigma_{r e f} \sigma_{m}-\sigma_{b}^{2}+\sigma_{r e f}^{2}=0
\end{aligned}
$$

In deep surface crack $\delta \leq \alpha$, so $\alpha>\frac{\sigma_{b}+\sigma_{r e f}}{\sigma_{r e f}(2-\beta)}$ must be satisfied. The normalized crack length expression for shallow cracks can be derived from Eq. (10) as Eq. (11) with the normalized depth, applied load and reference stress are known.

$$
\beta=\frac{-\Delta \pm \sqrt{\Delta^{2}+\alpha^{2}\left(\frac{2}{3} \sigma_{m} \sigma_{r e f}+\sigma_{b}^{2}-\sigma_{r e f}^{2}\right)}}{\alpha^{2} \sigma_{r e f}}
$$

where
$\Delta=\alpha \sigma_{b}-\sigma_{b}-\sigma_{r e f}+\alpha \sigma_{r e f}-\alpha^{2}+\frac{1}{3} \sigma_{m}$

In these two formulas, considering load conditions and the actual physical meaning of the surface deep crack, the sign before the square must be forsook, and the + signis the real root of equations (10). When $\sigma_{\text {ref }}$ reaches the yield limit $\sigma_{y}$, the calculated crack size is the limit damage of this structure, the expression is as follow.

$\beta=\frac{-\Delta \pm \sqrt{\Delta^{2}+\alpha^{2}\left(\frac{2}{3} \sigma_{m} \sigma_{y}+\sigma_{b}^{2}-\sigma_{y}^{2}\right)}}{\alpha^{2} \sigma_{y}}$

and $\alpha>\frac{\sigma_{b}+\sigma_{y}}{\sigma_{y}(2-\beta)}$

\section{COMPUTE INSTANCE AND EXPERIMENTAL VERIFICATION}

\subsection{Compute Instance}

Taking 7075T6 aluminum alloy plate for example, its main ingredients are as follows: $\mathrm{W}(\mathrm{Cu})=1.6 \%, \mathrm{~W}(\mathrm{Mg})=$ $2.5 \%, \mathrm{~W}(\mathrm{C} \mathrm{r})=0.23 \%, \mathrm{~W}(\mathrm{Zn})=5.6 \%$, the remaining is $\mathrm{Al}$. The elastic modulus of $7075 \mathrm{~T} 6$ is $71 \mathrm{GPa}$ and yield strength бy $378 \mathrm{MPa}$. Plugging the yield strength of 7075T6 aluminum alloy $\sigma_{y}=378 \mathrm{Mpa}$ in Eq. (6), Eq. (13) and boundary curve $\quad \alpha=\frac{\sigma_{b}+\sigma_{y}}{\sigma_{y}(2-\beta)} \quad$. In order to simplify calculations and convenient experimental verification replacing the value of $\sigma_{m}$ as $300 \mathrm{Mpa}, 200 \mathrm{Mpa}$ and $100 \mathrm{Mpa}$ respectively and set $\sigma_{b}=0$. Using these datums, the damage tolerances can be calculated from Eq. (6) and Eq. (13), these damage tolerance curves are shown in Fig. (3) of which $\alpha$ ranges from 0.1 to 1 with every 0.01 taking a value. The boundary points for shallow crack under these loads are $\alpha=0.7354, \alpha=0.8236$ and $\alpha=0.9118$.

As is shown in Fig. (3a), the left parts of the 3 respective curves which are broken by the boundary points are the limit crack sizes of shallow crack for 7075 aluminum alloy plate under the corresponding load, while the right parts are the limit crack sizes of deep crack for 7075 aluminum alloy plate under the corresponding load. The abscissa of this figure is the normalized limit depth of the crack, and the ordinate is the normalized limit crack width. The dashed area in this figure means the security crack size range of shallow crack in 7075 aluminum alloy plate under $300 \mathrm{MPa}$. From Fig. (3), not only the relationship between damage tolerance characteristics and loads could be easily obtained, but the relationship between limit depth and limit width of crack under a particular load could also be obtained, which could be used to judge whether the cracked crack size is secure under the load. For example, when the load $\sigma_{m}$ is $300 \mathrm{MPa}$ and the normalized crack depth $\alpha$ is 0.2 , the corresponding 
normalized limit of the crack width $\beta$ is 1.270 , however, the maximum normalized crack width in actual structure is 1 , and it means that the structure is safe under this condition; when the load $\sigma_{m}$ is $300 \mathrm{MPa}$ and the normalized crack depth $\alpha$ is 0.5 , the corresponding normalized limit of the crack width $\beta$ is 0.6981 , if the normalized crack width in actual structure is less than 0.6981 , the structure is safe, otherwise fracture occurs. In the meantime, normalized crack width could be taken as known quantity, the status of structure also can be judged by solving normalized crack depth. Thus, this figure could serve as reference for the health detection and maintenance time determination of the structure.

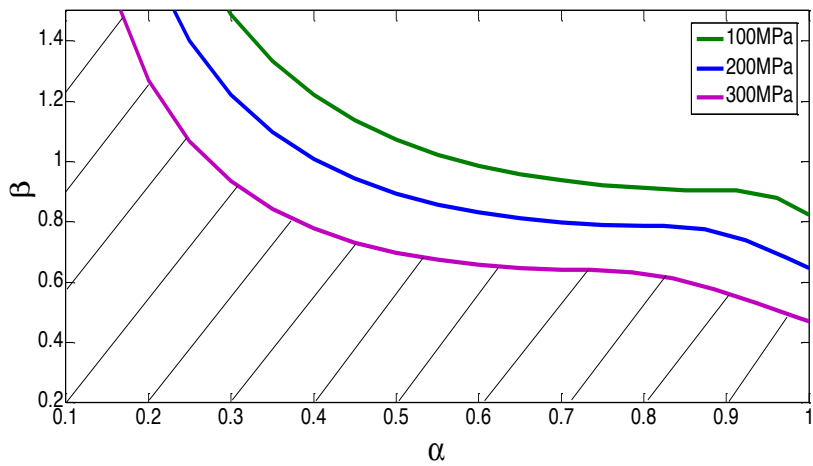

Fig. (3). Damage tolerance curve of 7075 aluminum alloy plate with crack under bending.

Meanwhile, as rated load in actual structure is constant, the rated load of cracked 7075 aluminum alloy plate $\sigma_{m}$ is assumed to be $300 \mathrm{Mpa}$. Normalized crack width $\beta$ is calculated using Eq. (6) and Eq. (10) with normalized crack depth $\alpha$ ranging from 0.1 to 1 and taking a value every 0.05 , then the damage tolerance curve (shown in Fig. 4) is obtained by fitting these data using rational polynomial approximation method and the regression equation of damage tolerance for cracked 7075 aluminum alloy plate under $300 \mathrm{MPa}$ bending load is:

$\beta=\frac{-2.985 \alpha^{4}+5.637 \alpha^{3}-3.484 \alpha^{2}+1.21 \alpha+0.007731}{\alpha-0.02717}$

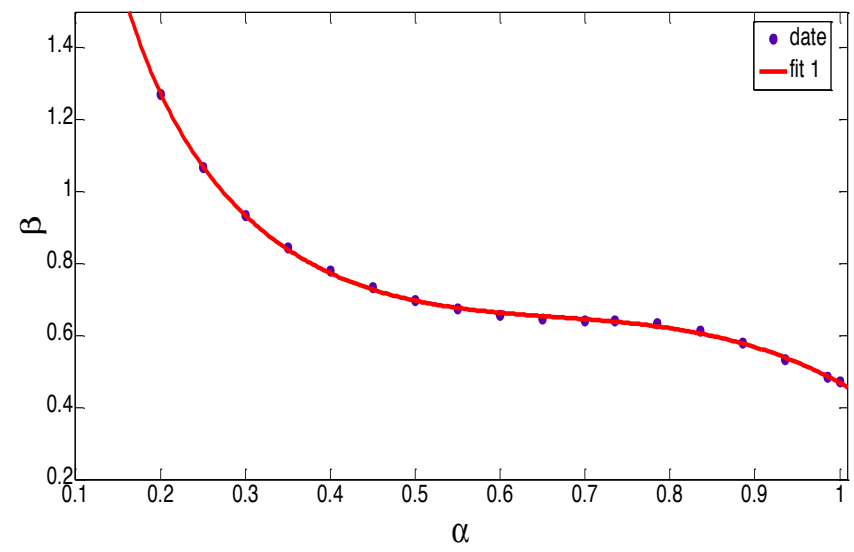

Fig. (4). The damage tolerance regression curve of cracked 7075 aluminum alloy.
The root mean square error of this regression equation is 0.005523. Using this method, regression model of damage tolerance for cracked 7075 aluminum alloy and other material plates under any loads also can be obtained.

\subsection{Experimental Verification}

According to GB3075-82 fatigue test method, the specimen is processed in a numerical control milling machine and its shape is shown in Fig. (1), the width $2 \mathrm{w}$ is 8 $\mathrm{mm}$, the length $2 \mathrm{~L}$ is $100 \mathrm{~mm}$ and the thickness $\mathrm{t}$ is $2.5 \mathrm{~mm}$. Different crack depths and widths are preseted in the center of the specimen by electro discharge machining method. The test is done on self-made vibration test platform, during which one edge of the specimen is held and the other is loaded cyclic loading by electromagnetic vibrator. Maximum excitation force of vibrator is $200 \mathrm{~N}$, and working frequency range from $0 \mathrm{~Hz}$ to $2000 \mathrm{~Hz}$. The selected frequency is $35 \mathrm{~Hz}$, the stress recycle ratio $\mathrm{R}$ is 0 and the maximum bending stress of crack section is $300 \mathrm{MPa}$. After the specimen crack, the sizes of crack propagation region and whole plastic crack region are measured by Wyko NT9100 produced by Veeco. The comparison of test results and the calculated results are shown in Figs. $(\mathbf{5}, \mathbf{6})$.

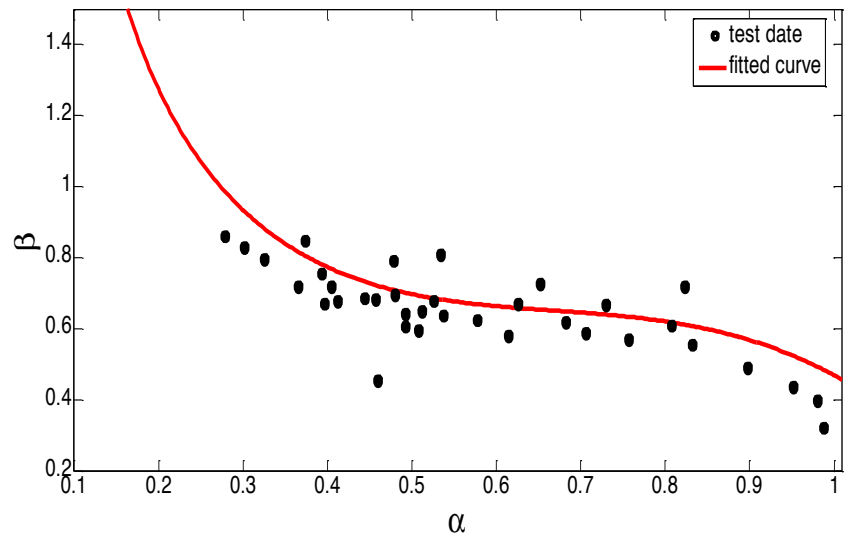

Fig. (5). The comparison of test results and the predicted results.

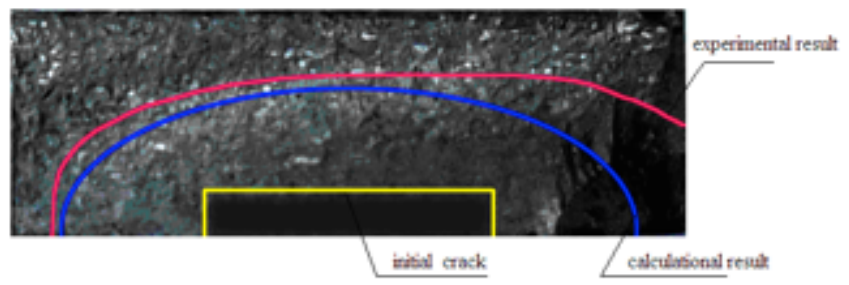

Fig. (6). the fractographic analysis of fractured test piece.

As shown in Fig. (5), although, there are some differences between the individual sample data and the results predicted by the model, the overall test results have good consistency with the results predicted by the model. The main reasons are the discreteness of material and microstructural such as local grain orientations and the machining error of initial crack, while the stress concentration generated by the surface scratch of specimen is another reason. It can be known from the fractographic analysis of fractured test piece, as is shown in Fig. (6), the experimental result agree well with the calculational result in the left part; but in the right part, the experimental result is slightly bigger than the calculational result. This is because 
there was a small crack generated by the stress concentration in this part during the experimental process.

\section{CONCLUSION}

(1) Based on the 'global' plastic collapse of the entire cross-section containing the defect, the damage tolerance prediction model was built by reference stress method, and then the model was verified by an experiment. The results show that the results predicted by the model are in good agreement with the test results during the shallow crack stage; while in the deep crack stage experimental results are slightly less than the predicted results

(2) The proposed model is not only applicable to cracked plate containing shallow crack to predict damage tolerance, but it also could be used to predict cracked plate containing a deep crack. This model could be used to give some reference to determine maintenance time and safety assessment of the cracked structure; however, taking the differences of individuals into consideration, at least $20 \%$ of safety margin should be set aside when using the model

\section{CONFLICT OF INTEREST}

The authors confirm that this article content has no conflict of interest.

\section{ACKNOWLEDGEMENTS}

This work is supported by supported by National Key Basic Research Development Plan of China "973" (No. 2010CB731703 and 2012CB619505).

\section{REFERENCES}

[1] M. Nakai, and T. Eto, "New aspects of development of high strength aluminum alloys for aerospace applications," Materials Science and Engineering A, vol. 285, pp. 62-68, June 2000.

[2] D. Feng-shan, Y. Liang, D. I. Sheng-long, and Y. Shou-jie. "Study on Fatigue Performance of High Strength Alumi- num Alloy,"
Journal of Aeronautical Materials, vol. 29, no. 1, pp. 96-100, Feb. 2009.

[3] Y. Liang, D. Feng-shan, D. Sheng-long, and Y. Shou-jie. "Effect of microstructures on fatigue crack propagation in 2E12 aluminum alloy," The Chinese Journal of Nonferrous Metals, vol. 20, no. 7, 1275-1281, Jul. 2010.

[4] I. Sattari-Far, and P. Dillstrom. "Local limit load solutions for surface cracks in plates and cylinders using finite element analysis," International Journal of Pressure Vessels and Piping, vol. 81, pp. 57-66, Jan. 2004.

[5] Y. Lei, "Use of local and global limit load solutions for plates with surface cracks under tension," International Journal of Pressure Vessels and Piping, vol. 84, pp. 545-559, Sep. 2007.

[6] Y. Lei, "J-integral and limit load analysis of semi-elliptical surface cracks in plates under combined tension and bending," International Journal of Pressure Vessels and Piping, vol. 81, pp. 43-56, Jan. 2004

[7] British Energy Generation Ltd, R6, assessment of the integrity of structures containing defects, revision 4, Gloucester, 2001. Amendment 8, 2010, pp. 8-20.

[8] K. Wasmer, K. M. Nikbin, and G. A. Webster, "Creep crack initiation and growth in thick section steel pipes under internal pressure," International Journal of Pressure Vessels and Piping, vol. 80, pp. 489-98, Jul. 2003.

[9] K. Wasmer, K. M. Nikbin, and G. A. Webster, "A sensitivity study of creep crack growth in plates to reference stress formulae," Proceeding of 2nd ECCC creep conference on creep \& fracture in high temperature componentse design \& life assessment issues, Zürich, CH, pp. 1294-1307, April 2009.

[10] R. A. Ainsworth, "Assessment of defects in structures of strain hardening material," Engineering Fracture Mechanics, pp. 633-42, 1984.

[11] A. J. Carter, "A Library of limit loads for fracture two, adopted as an option within the software programme R5-CODE, version 3.0, user guide," British Energy Generation Ltd, pp.89-120, 1999.

[12] K. Wasmer, K. M. Nikbin, and G. A. Webster, "Creep crack initiation and growth in thick section steel pipes under internal pressure," International Journal of Pressure Vessels and Piping, vol. 80, pp. 489-98, Jul. 2003.

[13] K. Wasmer, K. M. Nikbin, and G. A. Webster, "A sensitivity study of creep crack growth in plates to reference stress formulae," Proceeding of 2nd ECCC creep conference on creep \& fracture in high temperature componentse design \& life assessment issues, Zürich, CH, pp. 1294-1307, April, 2009.

[14] K. Wasmer, "Creep crack growth prediction in a range of steels," Mechanical Engineering, Imperial College, London, pp.25-30, July 2003. 